\title{
EGCG inhibits recepteur d'origine nantais expression by suppressing Egr-1 in gastric cancer cells
}

\author{
JUNG SUN PARK ${ }^{1}$, PHAM NGOC KHOI ${ }^{1}$, YOUNG EUN JOO ${ }^{1}$, YOUNG HAN LEE ${ }^{2}$, \\ SVEN A. LANG $^{3}$, OLIVER STOELTZING ${ }^{4}$ and YOUNG DO JUNG ${ }^{1}$ \\ ${ }^{1}$ Research Institute of Medical Sciences, Chonnam National University Medical School, Gwangju; \\ ${ }^{2}$ Department of Biomedical Science and Technology, SMART Institute of Advanced Biomedical Science, \\ RCTC, Konkuk University, Seoul, Republic of Korea; ${ }^{3}$ Department of Surgery, University Medical \\ Center Regensburg, Regensburg; ${ }^{4}$ Department of Hepatobiliary and Transplant Surgery, \\ University Medical Center Hamburg-Eppendorf, Hamburg, Germany
}

Received November 6, 2012; Accepted December 19, 2012

DOI: 10.3892/ijo.2013.1775

\begin{abstract}
Abnormal accumulation and activation of the recepteur d'origine nantais (RON) has been implicated in epithelial tumor carcinogenesis. In the present study, we examined the effect of epigallocatechin-3-gallate (EGCG), the major green tea catechin, on the induction of RON and tumor growth in human gastric cancer. EGCG inhibited phorbol 12-myristate 13-acetate (PMA)-induced RON expression and reduced RON transcriptional activity. However, (-)-epigallocatechin (EGC),(-)-epicatechin gallate (ECG) and (-)-epicatechin (EC) did not affect RON expression. Experiments with deleted and site-directed mutagenesis of the RON promoter indicated that Egr-1 binding sites in the RON promoter may be the EGCG-response element acting as a cis-element in gastric cancer cells. EGCG also inhibited PMA-induced Egr-1 expression and DNA binding in a dose-dependent manner. Furthermore, gastric cancer cells pretreated with PMA showed markedly enhanced invasiveness, which was partially abrogated by EGCG and siRNA-targeted RON and Egr-1. EGCG significantly reduced tumor growth in an in vivo tumor model, whereas RON expression was downregulated. These results suggest that EGCG may exert at least part of its anticancer effect by controlling RON expression through suppression of Egr-1 activation.
\end{abstract}

\section{Introduction}

Epidemiological and preclinical studies have reported that green tea consumption reduces the risk of cancer development $(1,2)$. The anticarcinogenic effects of green tea have been

Correspondence to: Dr Young Do Jung, Department of Biochemistry, Chonnam National University Medical School, 5 Hakdong, Gwangju 501-190, Republic of Korea

E-mail: ydjung@chonnam.ac.kr

Key words: epigallocatechin-3-gallate, recepteur d'origine nantais, Egr-1, gastric cancer attributed to the biological activities of its polyphenol components. Green tea extract contains (-)-epigallocatechin gallate (EGCG), (-)-epigallocatechin (EGC), (-)-epicatechin gallate (ECG) and (-)-epicatechin (EC) (3). EGCG, the most abundant polyphenol in green tea, inhibits cell proliferation and induces apoptosis in tumor cells (4). In addition to its cancer chemopreventive activity, EGCG inhibits tumor invasion, which is a crucial step in the metastasis of all solid tumors. In a previous study, we demonstrated that treating mice with EGCG resulted in marked inhibition of vascularity and proliferation of human colon cancer xenografts in nude mice (5); however, the mechanisms involved have yet to be fully elucidated.

Although the incidence rates of gastric cancer have declined in several industrialized countries over the past few decades, gastric cancer remains the most common cancer of the digestive tract, with a poor prognosis and high mortality rate (6). Due to local tissue invasion and metastasis, radiation therapy and chemotherapy do not significantly affect the survival or the quality of life of patients with advanced gastric cancer. Thus, developing effective therapeutic strategies against gastric cancer could help improve treatment strategies.

Recepteur d'origine nantais (RON), a member of the c-MET family of scatter factor receptors, plays an important role in the occurrence, progression and metastasis of gastric cancer (7). RON is activated through ligand-dependent or -independent mechanisms, which lead to responses associated with tumor development and metastasis (8). Macrophage-stimulating protein is the only RON ligand that has been identified thus far (9). Upon ligand binding, RON dimerizes, autophosphorylates and transduces a variety of signals that regulate different downstream pathways, including Ras/mitogen-activated protein kinase (MAPK), phosphatidylinositol 3-kinase, c-Jun $\mathrm{N}$-terminal kinase, $\beta$-catenin and nuclear factor- $\kappa \mathrm{B}(10,11)$. Several human tumor tissues exhibit aberrant expression and activation of RON, including tumors of the breast, colon and prostate gland (12). It has been suggested that multiple regulatory elements are required for full RON promoter activity and gene expression (13). Since RON plays a central role in multiple processes involved in cancer progression and metastasis, it is an attractive target for molecular-based cancer therapy. 
In this study, we discovered that EGCG suppressed RON expression in cancer cells and inhibited tumor growth in vivo. We verified the EGCG-response elements in the RON promoter in order to investigate the mechanism behind EGCG-mediated regulation of RON.

\section{Materials and methods}

Cell culture and culture conditions. AGS human gastric cancer cells were obtained from the American Type Culture Collection (Manassas, VA, USA) and MKN28 cells were obtained from the Korean Cell Line Bank (Seoul, Korea). The TMK-1 human gastric cancer cell line was provided by Dr Eiichi Tahara (Hiroshima University, Hiroshima, Japan). The cells were cultured in RPMI-1640 supplemented with $10 \%$ fetal bovine serum (FBS) and $1 \%$ penicillin-streptomycin at $37^{\circ} \mathrm{C}$ in an atmosphere containing $5 \% \mathrm{CO}_{2}$. AGS cells pretreated with $30 \mu \mathrm{M}$ EGCG for $1 \mathrm{~h}$ were exposed to $200 \mathrm{nM}$ phorbol 12-myristate 13-acetate (PMA) for $8 \mathrm{~h}$ and the levels of RON were analyzed by western blot analysis to determine the effect of EGCG on the tumor promoter.

Western blot analysis. Cells were suspended in ice-cold RIPA-M buffer with 1\% NP-40 and cell lysates were prepared as previously described (7). Cell lysate proteins (100 $\mu \mathrm{g})$ were resolved on $10 \%$ sodium dodecyl sulfate-polyacrylamide gel electrophoresis and transferred to nitrocellulose membranes (Bio-Rad, Hercules, CA, USA). The blots were blocked for at least $1 \mathrm{~h}$ at room temperature in blocking buffer (5\% non-fat dry milk in Tris-buffered saline containing 0.05\% Tween-20; TBST). Anti-RON $\beta$ (Santa Cruz Biotechnology, Santa Cruz, CA, USA) was diluted in blocking buffer and incubated with the blots overnight at $4^{\circ} \mathrm{C}$. The bound antibodies were detected with a 1:3,000 dilution of horseradish peroxidase-conjugated secondary antibody, according to the instructions of the enhanced chemiluminescence kit (Amersham, Franklin Lakes, NJ, USA).

Construction of the RON promoter-reporter construct. A construct of the RON promoter fragment, $3 \mathrm{~kb}$ in length, was synthesized from human genomic DNA (Promega, Madison, WI, USA) by polymerase chain reaction (PCR) using the primers 5'-GGTACCTAGCTGACC-3' (forward) and 5'-GGGCCAAATTTAAGC-3' (reverse). The amplified PCR products were ligated into the T\&A Vector (RBC Bioscience, Saskatoon, SK, Canada), then digested with $K p n I$ and $B g l I I$. The products were ligated into the KpnI and $B g l$ II sites of the pGL3-Basic Vector (Promega). A series of deletion constructs of the human RON promoter fragments was synthesized by PCR using the pRON-Luc plasmid as the template. The forward primer sequences were: 5'-CCAAGGGCCGGAAGA-3' (-128/+173, pGL3-RON-301), 5'-TCGGCTGAGCGCTAA-3' (-20/+173, pGL3-RON-193) and 5'-TCGTGCGTCCGCAGG-3' (+50/+173, pGL3-RON-123). One reverse primer, 5'-GGGCCA AATTTAAGC-3', was used to generate all three deletion constructs. The amplified PCR products were ligated into the T\&A Vector and then digested with $K p n I$ and $B g l$ II. Subsequently, the products were ligated into the KpnI and BglII sites of the pGL3-Basic Vector. Site-directed mutagenesis was utilized to mutate potential transcriptional Egr-1 elements in the promoter region. Mutant promoter constructs were generated using the pGL3-RON-301 construct as a template. The primers used for mutagenesis (mutations underlined) were: TCC $\underline{\text { GCCGCC }}$ to TCCATATGCC (pGL3-Mt1) and CCCGCCCCCA to CCCAATTCCA (pGL3-Mt2). The mutated nucleotide sequences of all mutant constructs were confirmed by DNA sequencing.

RON promoter-reporter assay. Transcriptional regulation of RON was examined by transient transfection of a RON promoter-luciferase reporter construct (pGL3-RON). Gastric cancer cells $\left(5 \times 10^{5}\right)$ were seeded and grown until they reached $60-70 \%$ confluence and pGL3-RON wild-type and deletion mutants were transfected into the cells using Lipofectamine $^{\mathrm{TM}} 2000$ (Invitrogen, Carlsbad, CA, USA), according to the manufacturer's protocol. The significance of the Egr-1 binding site as an EGCG-response element in the regulation of RON was examined by co-transfecting the cells with pGL3-RON and Egr-1 expression plasmids containing full-length complementary DNA (cDNA) coding for human Egr-1 (a gift from Dr Young Han Lee, Konkuk University, Seoul, Korea). The pRL-null plasmid encoding Renilla luciferase was included in all the samples to monitor transfection efficiency. At $24 \mathrm{~h}$ post-transfection, the levels of firefly and Renilla luciferase activity were measured sequentially from a single sample using the Dual-Glo Luciferase Assay system (Promega). Firefly luciferase activity was normalized to Renilla activity and the relative amount of luciferase activity in the untreated cells was designated as 1 .

Reverse transcription-PCR. Total RNA was extracted from AGS cells using TRIzol reagent (Invitrogen). One microgram of total RNA was used for first-strand cDNA synthesis using random primers and superscript reverse transcriptase (Invitrogen). The cDNA was subjected to PCR amplification with the Egr-1 and glyceraldehyde 3-phosphate dehydrogenase (GAPDH) primer sets. The specific primers sequences were: $E g r$-1, sense, 5'-CAGTGGCCTAGTGAGCATGA-3' and antisense, 5'-CCGCAAGTGGATCTTGGTAT-3' (786 bp); GAPDH, sense, 5'-TTGTTGCCATCAATGACCCC-3' and antisense, 5'-TGACAAAGTGGTCGTTGAGG-3' (836 bp). The PCR conditions were: denaturation at $94^{\circ} \mathrm{C}$ for $20 \mathrm{sec}$, annealing at $53^{\circ} \mathrm{C}$ for $20 \mathrm{sec}$ and extension at $72^{\circ} \mathrm{C}$ for $50 \mathrm{sec}$.

Chromatin immunoprecipitation assay. AGS cells $\left(2 \times 10^{6}\right)$, grown in 6-well plates, were cross-linked with $0.5 \%(\mathrm{v} / \mathrm{v})$ formaldehyde at $37^{\circ} \mathrm{C}$ for $5 \mathrm{~min}$. The cells were sonicated for $3 \times 20 \mathrm{sec}$ prior to centrifugation at $16,000 \mathrm{x} \mathrm{g}$ for $15 \mathrm{~min}$ at $4^{\circ} \mathrm{C}$. The specific DNA-bound transcription factor complexes were precipitated with $20 \mu \mathrm{l}$ anti-Egr- 1 at $4^{\circ} \mathrm{C}$ overnight prior to the addition of Protein $\mathrm{A}$ agarose beads. The proteins were removed from the DNA by digestion with $10 \mu \mathrm{g} / \mathrm{ml}$ Proteinase $\mathrm{K}$ at $65^{\circ} \mathrm{C}$ for $30 \mathrm{~min}$. The DNA was recovered from the solution using the QIAquick PCR Purification kit (Qiagen Inc., Valencia, CA, USA) and eluted in $50 \mu 1$ sterile water. Eluted DNA $(20 \mu \mathrm{l})$ was subjected to PCR with forward, -5'-AGGAGCCAGGCCTCCAAGGGC-3' and reverse, -5'-TCCCGACAGCCCCAAGATAGC-3' primers, which flank the Egr-1 binding sites. 
Small interfering RNA transfection. Gene silencing was performed using human Egr-1 (sc-29303; Santa Cruz Biotechnology) and human RON sequence-specific duplex small interfering RNA (siRNA) (sc-36434). Briefly, $20 \mathrm{nM}$ of siRNA oligonucleotides and $2 \mu \mathrm{l}$ of Lipofectamine RNAiMAX (Invitrogen) were mixed with $100 \mu \mathrm{l}$ of Opti-MEM serum-free medium (Hyclone, Logan, UT, USA) for each transfection reaction in two separate tubes and incubated for $5 \mathrm{~min}$ at room temperature. Subsequently, the contents of the two tubes were combined and allowed to form siRNA-Lipofectamine complexes for $30 \mathrm{~min}$ at room temperature. A $900 \mu \mathrm{l}$ volume of AGS cells cultured in serum-free medium was combined with the siRNA-Lipofectamine mix, plated in the wells of a 6-well tissue culture dish and placed in a $37^{\circ} \mathrm{C}, 5 \% \mathrm{CO}_{2}$ incubator for $5 \mathrm{~h}$. The medium was replaced with normal growth medium.

Matrigel invasion assay. The cell invasion assay was carried out using BioCoat Matrigel Invasion Chambers (Becton-Dickinson, Bedford, MA, USA) with 10\% FBS as the chemoattractant in the lower chamber. AGS cells $\left(10^{5}\right)$ in $300 \mu \mathrm{l}$ were allowed to invade the Matrigel for $24 \mathrm{~h}$. The noninvading cells on the upper surface of each membrane were removed from the chamber and the invading cells on the lower surface of each membrane were stained with the Diff-Quick Stain kit (Becton-Dickinson). Following two washes with water, the chambers were allowed to air-dry. The number of invading cells was counted using a phase-contrast microscope.

Animal model. Eight-week-old male athymic nude mice (BALB/cnu/nu; Charles River, Sulzfeld, Germany) were used for the experiments, as approved by the Institutional Animal Care and Use Committee of the University of Regensburg and the regional authorities. Experiments were conducted according to the Guidelines for the Welfare of Animals in Experimental Neoplasia by the United Kingdom Coordinating Committee on Cancer Research. The effects of EGCG inhibition on the growth of TMK-1 human gastric cancer cells were investigated in a subcutaneous xenograft tumor model. TMK-1 cells $\left(1 \times 10^{6}\right)$ were injected subcutaneously into the right flank of nude mice. Mice were randomized ( $\mathrm{n}=8$ per group) and assigned to treatment groups. Intraperitoneal injections of EGCG $(1 / \mathrm{mg} / \mathrm{mouse}$, twice/week) were initiated on Day 1 . The control group $(n=8)$ was treated with the same dosage of EC. Tumor diameters were measured every other day and tumor volumes were calculated (width ${ }^{2} \mathrm{x}$ length $\mathrm{x} 0.5$ ). The experiment was terminated on Day 27 following tumor cell injection, and the tumors were then excised, weighed and prepared for western blot analyses.

\section{Results}

Effect of EGCG on PMA-induced RON expression in gastric cancer cells. To examine whether green tea catechins inhibit PMA-induced RON expression in gastric cancer cells, AGS human gastric carcinoma cells were pretreated with $30 \mu \mathrm{M}$ catechins for $1 \mathrm{~h}$ prior to an 8 -h incubation with $200 \mathrm{nM}$ of PMA, and the RON protein levels were measured by western blot analysis. EGCG at $30 \mu \mathrm{M}$ inhibited PMA-induced RON protein expression. However, other tea catechins such as EC, ECG and EGC inhibited RON expression only slightly at the same concentrations (Fig. 1A). We then examined the effect
A

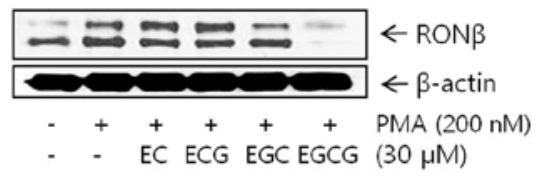

B

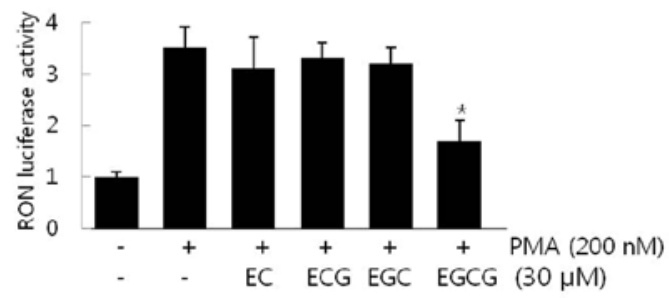

C

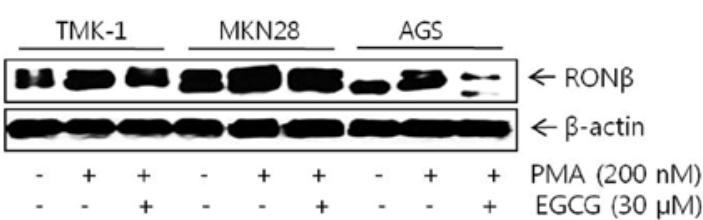

D

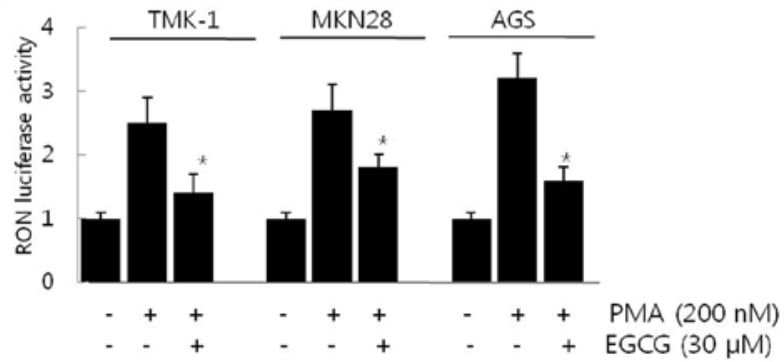

Figure 1. Effect of EGCG on PMA-induced RON expression in gastric cancer cells. (A) AGS cells were exposed to $200 \mathrm{nM}$ of PMA for $8 \mathrm{~h}$ and the levels of RON were determined by western blot analysis following pretreatment with $30 \mu \mathrm{M}$ of EC, ECG, EGC and EGCG for $1 \mathrm{~h}$. (B) AGS cells were transiently transfected with the pGL3-RON reporter (pRON-Luc, -128/+173) construct. Following pretreatment with $30 \mu \mathrm{M}$ of EC, ECG, EGC and EGCG for $1 \mathrm{~h}$, the transfected cells were exposed to $200 \mathrm{nM}$ of PMA for $8 \mathrm{~h}$ and luciferase activity was determined using a luminometer. (C) Following pretreatment with $30 \mu \mathrm{M}$ of EGCG for $1 \mathrm{~h}$, TMK-1, MKN28 and AGS cells were exposed to $200 \mathrm{nM}$ of PMA for $8 \mathrm{~h}$ and the levels of RON were determined by western blot analysis. (D) TMK-1, MKN28 and AGS cells were transiently transfected with the pGL3-RON reporter (pRON-Luc, -128/+173) construct. Following pretreatment with $30 \mu \mathrm{M}$ of EGCG for $1 \mathrm{~h}$, the transfected cells were exposed to $200 \mathrm{nM}$ of PMA for $8 \mathrm{~h}$ and luciferase activity was determined using a luminometer. Data are the means \pm standard deviation from triplicate measurements. ${ }^{*} \mathrm{P}<0.05$ vs. PMA.

of EGCG on transcriptional regulation of the RON gene induced by PMA. AGS cells were transiently transfected with the promoter-reporter construct (pGL3-RON) of the human RON gene fused to the luciferase gene. AGS cells transfected with pGL3-RON exhibited an $\sim 3$.5-fold increase in promoter activity following PMA treatment (Fig. 1B). When the transfected cells were pretreated with $30 \mu \mathrm{M}$ catechins prior to PMA treatment, only EGCG significantly inhibited PMA-induced RON promoter activity (Fig. 1B). Catechins at the same concentrations did not affect cell viability (data not shown). TMK-1, MKN28 and AGS gastric cells were used to explore whether EGCG was able to inhibit RON expression in various gastric cancer cells. As shown in Fig. 1C and D, EGCG inhibited PMA-induced RON expression and promoter 
A

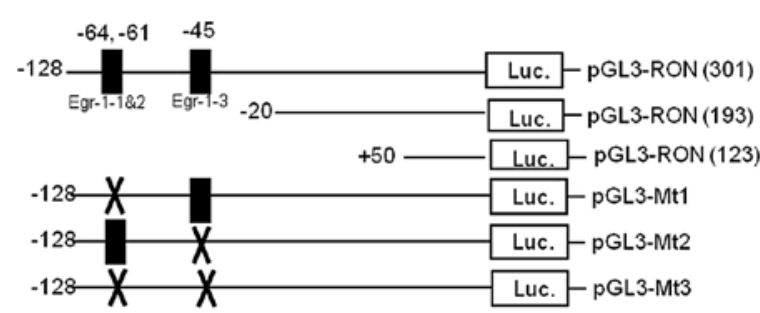

B

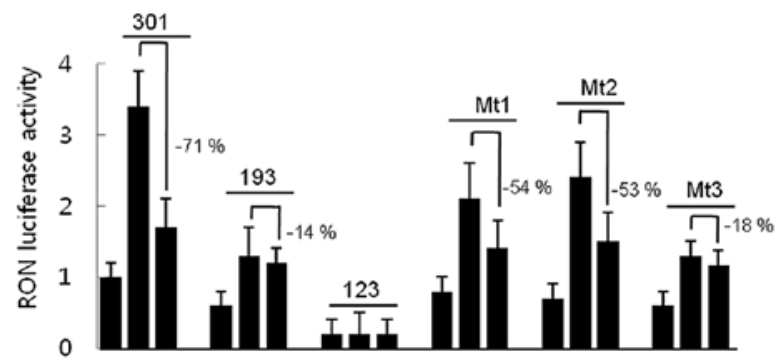

Figure 2. EGCG-response elements in the RON promoter in gastric cancer cells. (A) Schematic representation of Egr-1 binding sites and deleted and mutated regions in the human RON promoter. (B) AGS cells were transiently transfected with a series of 5'-deletion constructs of the RON promoter-reporter plasmid or site-specific mutants for Egr-1 (Mt1, Mt2 and Mt3) derived from the $-128 /+173$ construct. Following pretreatment with $30 \mu \mathrm{M}$ of EGCG for $1 \mathrm{~h}$, the transfected cells were exposed to $200 \mathrm{nM}$ of PMA for $8 \mathrm{~h}$ and luciferase activity was determined using a luminometer. Data are the means \pm standard deviation from triplicate measurements.

activity in TMK-1, MKN28 and AGS cells. Collectively, these results demonstrate that EGCG suppressed the expression of the RON gene and reduced its promoter activity in human gastric cancer cells.

EGCG-response elements in the gastric cancer cell RON promoter. Promoter deletion analyses were performed to locate cis-response elements in the RON promoter in response to EGCG, in order to explore the underlying molecular mechanisms by which EGCG reduced RON gene promoter activity in AGS gastric cancer cells. AGS cells were transfected with pGL3-RON promoter-reporter constructs of different lengths (Fig. 2A). AGS cells were pretreated with $30 \mu \mathrm{M}$ of EGCG for $1 \mathrm{~h}$ prior to an 8 -h incubation with $200 \mathrm{nM}$ of PMA, and luciferase activity was measured. As shown in Fig. 2B, EGCG significantly reduced luciferase activity by $71 \%$ in cells transfected with pGL3-RON (301). However, cells transfected with pGL3-RON (193) resulted in a decrease of $14 \%$ in response to EGCG, suggesting that the -128 to -20 DNA fragment in the RON promoter might contain the EGCG-response element(s). A computer-aided search revealed three consensus Egr-1 binding sites in the -128 to -20 DNA fragment of the RON promoter. Site-directed mutagenesis of the Egr-1 binding site was generated in the pGL3-RON plasmids (301) to examine the role of the Egr-1 binding site in the inhibition of RON promoter activity by EGCG (Fig. 2A). A construct containing a mutation in the core sequence of the Egr-1-1 and -2-binding motif (CCCGCCCCCA to CCCAATTCCA, pGL3-Mt1) and the Egr-1-3-binding motif (TCCGCCCGCC to TCCATATGCC, pGL3-Mt2) reduced PMA responsiveness
A

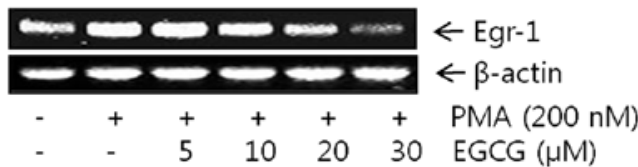

B
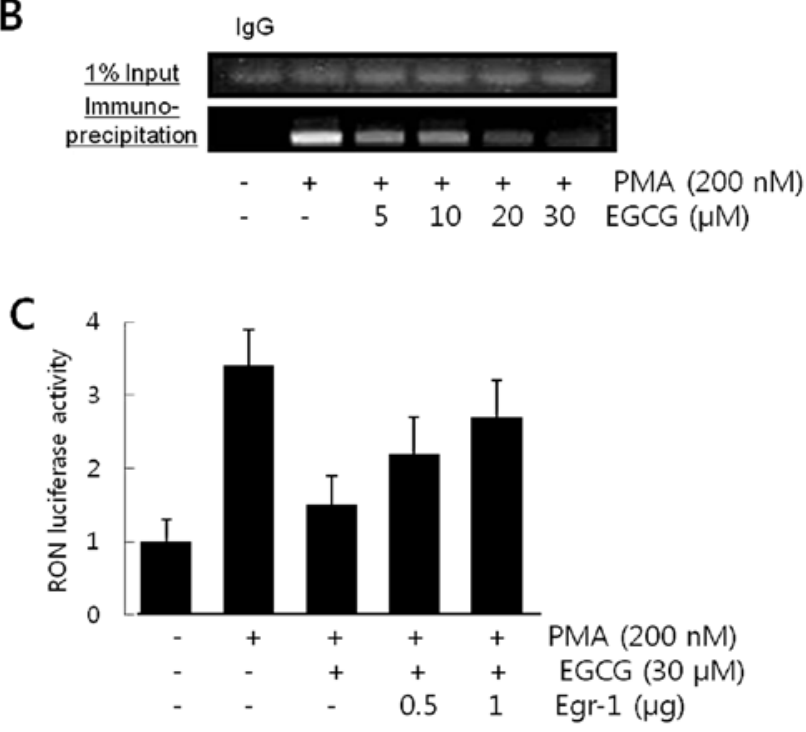

Figure 3. The role of Egr-1 in the inhibition of RON by EGCG in gastric cancer cells. (A) Following pretreatment with $30 \mu \mathrm{M}$ of EGCG for $1 \mathrm{~h}$, AGS cells were exposed to $200 \mathrm{nM}$ of PMA for $8 \mathrm{~h}$ and Egr-1 mRNA levels were determined by RT-PCR. (B) A chromatin immunoprecipitation assay was performed with anti-Egr-1 antibodies at the RON promoter in cells treated with EGCG. Following pretreatment with $0-30 \mu \mathrm{M}$ of EGCG for $1 \mathrm{~h}$, the AGS cells were exposed to $200 \mathrm{nM}$ of PMA for $8 \mathrm{~h}$ and were cross-linked, lysed and immunoprecipitated with anti-Egr-1 along with normal rabbit IgG (negative control). The precipitated DNA was subjected to regular PCR with primers specific for Egr-1 (-407/-112). (C) AGS cells were transiently cotransfected with the pGL3-RON reporter (pRON-Luc, -128/+173) construct and different concentrations of Egr-1 expression plasmids. Following pretreatment with $30 \mu \mathrm{M}$ of EGCG for $1 \mathrm{~h}$, the transfected cells were exposed to $200 \mathrm{nM}$ of PMA for $8 \mathrm{~h}$ and luciferase activity was determined using a luminometer. Data are the means \pm standard deviation from triplicate measurements.

to $\sim 54$ and $53 \%$, respectively, of that of the wild-type construct. However, a double-mutant promoter, containing mutations in Egr-1-1 and -2, as well as in Egr-1-3 (pGL3-Mt3), resulted in an $18 \%$ decrease in the response to EGCG, indicating that the presence of the wild-type Egr-1 binding sites was required for EGCG to reduce RON promoter activity (Fig. 2B). Collectively, these results suggest that the Egr-1 binding sites in the RON promoter might be the EGCG-response element acting as a cis-element to regulate promoter activity.

Role of Egr-1 in the inhibition of RON by EGCG in gastric cancer cells. Egr-1 mRNA level was determined by RT-PCR to verify the inhibitory mechanisms of EGCG on Egr-1 activity in AGS gastric cancer cells. As shown in Fig. 3A, EGCG dosedependently reduced PMA-induced Egr-1 expression at the transcriptional level. A chromatin immunoprecipitation assay was performed to further determine whether EGCG inhibits Egr-1 binding to the putative Egr-1-binding sequence in the RON promoter. AGS cell chromatin was immunoprecipitated 
A

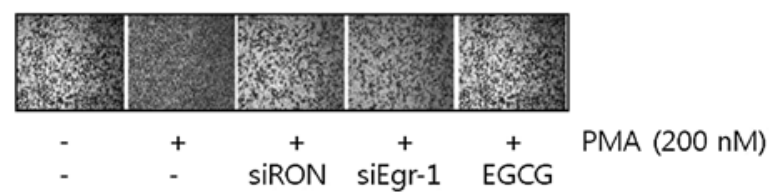

B

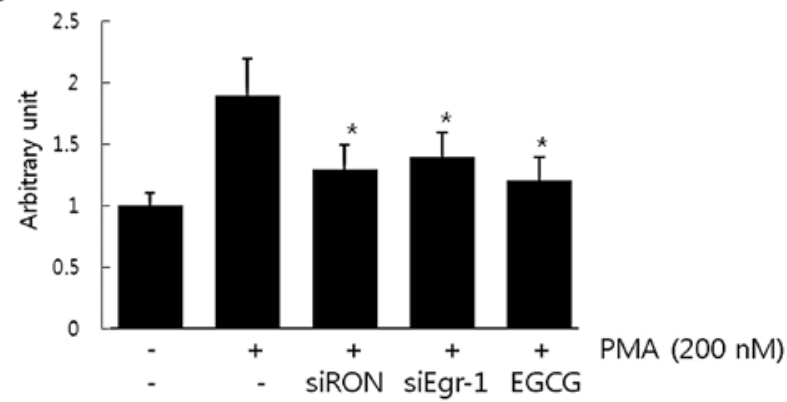

Figure 4. Effect of siRON, siEgr-1 and EGCG on PMA-induced gastric cancer cell invasion. AGS cells $\left(10^{5}\right)$, transfected with RON siRNA and Egr-1 siRNA or pretreated with $30 \mu \mathrm{M}$ of EGCG, were incubated with $200 \mathrm{nM}$ of PMA in a Boyden Matrigel apparatus for $24 \mathrm{~h}$. (A) Following incubation, the cells that invaded the undersurface of the chambers were counted using a phase-contras light microscope after staining with a Diff-Quick Stain kit. (B) Data are the means \pm standard deviation from triplicate measurements. ${ }^{*} \mathrm{P}<0.05$ vs. PMA.

with rabbit anti-Egr-1 antibody and the resulting immunoprecipitates were analyzed by PCR using primers flanking the Egr-1-binding sequences (-407 to -112) of the RON promoter. An evident increase in DNA band intensity was observed in cells treated with PMA and anti-Egr-1 antibody, but not when normal rabbit IgG was used (Fig. 3B). When the cells were pretreated with 0-30 $\mu \mathrm{M}$ of EGCG prior to PMA treatment, the induction of Egr-1-DNA binding by PMA was inhibited in a dose-dependent manner (Fig. 3B). To explore the role of Egr-1 in regulating the RON promoter activity, AGS cells were co-transfected with the RON promoter-luciferase reporter and the Egr-1 cDNA expression plasmid (pEgr-1cDNA) at the indicated concentrations. As shown in Fig. 3C, forced expression of Egr-1 cDNA dose-dependently eliminated the EGCG inhibitory effect, suggesting that the increase in the abundance of cellular Egr-1 eradicated the inhibitory effect of EGCG on RON promoter activity in AGS cells.

Effect of EGCG, siRON and siEgr-1 on PMA-induced gastric cancer cell invasion. It has been suggested that RON expression is essential for the invasive phenotype of cancer cells. The role of PMA-induced RON in AGS cell invasion was evaluated in a modified Boyden invasion chamber. As shown in Fig. 4, cell invasiveness increased 2 -fold following incubation with PMA. However, cells transfected with RON siRNA and Egr-1 siRNA partially lost the Matrigel invasiveness induced by PMA. These results suggest that PMA-induced Egr-1 and RON in AGS cells stimulated AGS cell invasion. In addition, the effect of EGCG on AGS cell invasion stimulated by PMA was examined. Cells pretreated with $30 \mu \mathrm{M}$ of EGCG also partially abrogated PMA-induced cell invasion. Our results suggest that treating AGS cells with EGCG reduced RON by regulating Egr-1 and that these events contributed to a reduction in AGS gastric cancer cell invasion.
A

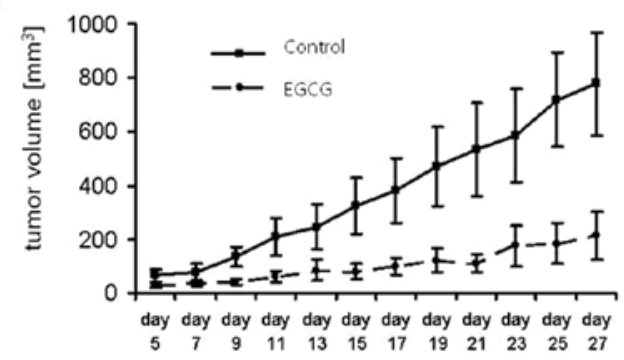

B
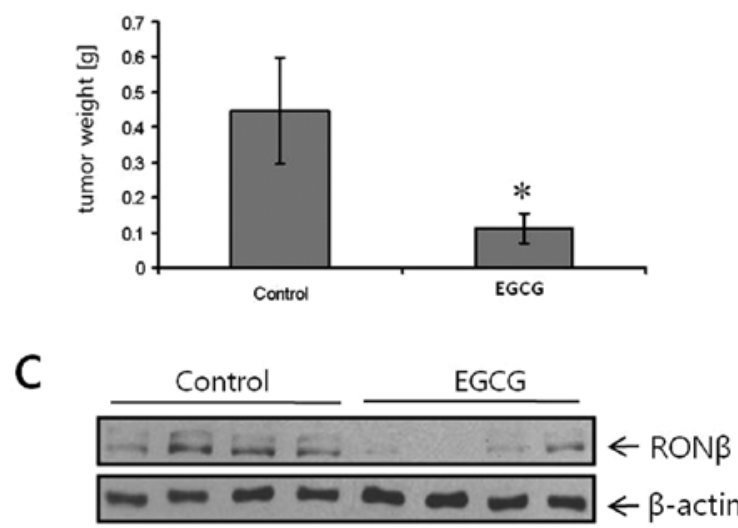

Figure 5. Effects of EGCG on tumor growth and RON expression in vivo. The effects of EGCG (1 mg/day/mouse, intraperitoneally) and EC (control) on (A) tumor growth and (B) tumor weight at 27 days following tumorcell implantation were investigated in a subcutaneous xenograft model ( $n=8 /$ group). Bars indicate standard error. $\mathrm{P}<0.05$ ( 8 mice/group). (C) RON levels in the gastric tumor tissues were determined by western blot analysis.

Effects of EGCG on tumor growth and RON expression in vivo. The effects of EGCG on cancer growth in vivo were determined in a subcutaneous gastric (TMK-1) cancer xenograft model. Treatment with EGCG (1 mg in 0.1-0.2 ml PBS/day/mouse) inhibited gastric tumor growth (Fig. 5A). The control group was treated with the same dosage of EC. The potent growthinhibitory effect was also mirrored by the weights of the excised tumors, which were significantly decreased in the EGCG-treated mice (Fig. 5B). Notably, in vivo RON expression levels were also substantially decreased in mice treated with EGCG, compared to controls (Fig. 5C). Mouse body weight did not differ among treatment groups (data not shown). We concluded that EGCG sufficiently inhibited gastric cancer cell growth in vivo. Our data provide the first evidence that the inhibition of tumor growth by EGCG may, in part, be mediated by impairing the RON system.

\section{Discussion}

We previously delineated the role of RON in the acquisition of the gastric cancer cell invasive phenotype and we also identified the critical regulatory elements that are necessary for oncogenic RON tyrosine kinase promoter activity and gene expression (14). Tyrosine kinase receptors regulate multiple processes involved in tumor progression and metastasis, making them attractive molecular therapy targets. RON is mainly transcribed at relatively low levels in normal epithelial cells. However, the levels of RON expression in malignant epithelial cells increase severalfold compared with those in 
benign epithelium. This aberrant expression and activation of RON has been observed in human cancer and is responsible for various malignant behaviors in breast, colon and ovary cancer $(12,15)$. Increased RON expression is strongly correlated with phosphorylation and tumor invasiveness, suggesting that increased RON expression plays a role in the progression of carcinomas to an invasive-metastatic phenotype (16).

In this study, we demonstrated that EGCG suppressed RON expression by regulating Egr-1 in gastric cancer cells and that these events may contribute to the reduction of tumor growth in vivo. Interest in green tea as an anticancer agent in humans has increased for several reasons: i) several epidemiological studies have reported that green tea lowers the risk of cancer, when consumed in large amounts (17); ii) green tea inhibits the development and progression of skin, lung, mammary gland and gastrointestinal tract cancer in animal models (18); iii) green tea extracts, including purified EGCG, stimulate apoptosis in various cancer cell lines, such as stomach, prostate, lymphoma and lung in vitro (5); and iv) green tea consumption is associated with few adverse events and it can be easily obtained at a low cost (19). The anticancer effects of green tea have been attributed to the biological activities of its polyphenol components. EGCG is the most abundant of the green tea polyphenols, accounting for $>40 \%$ of the total polyphenolics (3). Several molecular mechanisms have been suggested for the observed anticancer effect of EGCG, including suppression of ligand binding to the epidermal growth factor receptor (EGFR) (20), inhibition of protein kinase C (21), lipoxygenase and cyclooxygenase activities (3), induction of apoptotic cell death and arrest of the tumor cell cycle (5).

The suppression of RON expression by EGCG occurred at the transcriptional level, as shown by the transient transfection study using the RON promoter-reporter construct (Fig. 1). It has previously been suggested that multiple regulatory elements are required for full RON promoter activity and a portion of the 5'-flanking region of the RON gene has been cloned (13). Similar to numerous tyrosine kinase receptor gene promoters, the RON promoter also lacks distinct TATA box and CCAAT sequences. However, it contains several GC boxes, seven Sp1-binding sites, four retinoblastoma control elements, three IL-6 response elements and two AP-2 elements (15). Putative Egr-1-binding motifs in the RON gene promoter region of gastric cancer cells have also been reported (14). The role of the Egr-1 signaling pathway in the inhibition of RON gene expression by EGCG was evaluated in the present study. Site-directed mutagenesis of Egr-1 binding sites resulted in a decrease in the effects of EGCG on RON expression (Fig. 2). In addition, forced Egr-1 expression eliminated the inhibitory effects of EGCG in a dose-dependent manner (Fig. 3). These results indicate that interruption of Egr-1 by EGCG played a critical role in suppressing RON gene promoter activity. A similar finding was reported by $\mathrm{Fu}$ and Chen (22), who observed that EGCG suppresses EGFR gene expression in rat hepatic stellate cells by reducing Egr-1 activity. They suggested that inhibition of Egr-1 transactivation activity and the EGFR gene promoter activity by EGCG occurs through an interruption in the ERK signaling pathway. By contrast, regarding the effect of EGCG on Egr-1 in human pulmonary epithelial cells, Moon et al reported that EGCG induced Egr-1 expression and mediated Egr-1 nuclear translocation via the ERK signaling pathway in pulmonary epithelial cells (23).
Comparing these findings with the downregulation of Egr-1 by EGCG demonstrated by our results, it can be hypothesized that Egr-1 and ERK responses are differentially regulated by EGCG, depending on the tissue environment and external stimuli.

The Egr-1 transcription factor is an immediate-early response gene that is rapidly induced by various growth factors, cytokines and DNA-damaging agents, and modulates cell proliferation, differentiation, apoptosis and inflammation in a variety of cells $(24,25)$. The role of Egr-1 in tumor development might be largely dependent on tissue type, since Egr-1 is highly expressed and plays an essential role in tumor growth and survival in various types of cancer $(26,27)$. The exact mechanisms by which EGCG inhibits the activation of Egr-1 are unknown. One possible explanation is that EGCG inhibits the kinases that are involved in the activation Egr-1. Numerous studies have suggested a causal relationship between ERK activation and induction of Egr-1 gene expression $(28,29)$. Activation of ERK induces Egr-1 gene expression mediated by the transcription factor Elk-1, an ERK substrate (29). Elk-1 binds to multiple serum response elements and their adjacent Ets motifs located in the Egr-1 gene promoter and stimulates promoter activity, leading to Egr-1 transcription (29). Previous studies suggested that MAPK is one of the target molecules in the signaling cascades regulated by EGCG. It was previously discovered that EGCG treatment suppresses ERK phosphorylation, resulting in the downregulation of vascular endothelial growth factor in HT29 human colon adenocarcinoma cells (5). Katiyar et al (30) demonstrated that pretreatment of human epidermal keratinocytes with EGCG inhibits ultraviolet $\mathrm{B}$-induced hydrogen peroxide production and hydrogen peroxide-mediated phosphorylation of the MAPK signaling pathway. EGCG modulates multiple signaling pathways, and the tyrosine kinase receptor (30), which is a strong metal ion chelator (31). Since certain kinases depend on divalent cations for their activity, EGCG may inhibit the activity of receptor kinases by chelating divalent cations. On the other hand, the EGCG inhibitory effects could be considered 'non-specific'. It has been demonstrated that EGCG non-specifically binds proteins and modulates enzyme activity, leading to inhibition of cell cycle-related kinases, MAPK and the activity of receptor tyrosine kinases (32). Our results do not exclude the role of other signaling pathways in EGCG-induced suppression of RON gene expression.

EGCG administration inhibits carcinogenesis in several animal models. Our results suggest, for the first time, that EGCG may exert its anticancer effects by inhibiting RON, supporting a role for green tea in cancer chemoprevention. The suitability of RON as a therapeutic target has been demonstrated in an experimental study that used a novel function-blocking antibody (33). The growth-inhibitory effects of the RON antibody have been validated in preclinical tumor models and a comprehensive analysis was performed on RON expression in various human cancer entities. The authors concluded that inhibiting RON is a potentially useful target for human cancer therapy. However, another report revealed that the growth-inhibitory effects of RON inhibitors may be transient (34). In that study, Logan-Collins et al demonstrated that silencing RON reduces tumor growth and renders cancer cells susceptible to chemotherapeutic agents; however, downregulation of RON is lost over time. This transient effect was linked 
to an increase in c-MET and EGFR expression, suggesting that these two oncogenic receptor systems provide an escape mechanism from RON silencing in cancer cells. The EGFR and c-MET findings are particularly relevant to our study, since both are possibly inhibited by EGCG. Thus, further studies are required to elucidate the detailed mechanism by which EGCG inhibits RON activity and to examine whether EGCG exerts the same effects in human cancer.

\section{Acknowledgements}

This study was supported by a research grant (0720570) from the National Cancer Center, by a Basic Science Research Program through the National Research Foundation of Korea (NRF) funded by the Ministry of Education, Science and Technology (2010-0009910) and by a Medical Research Center grant (2012-000-9442) from the Korean Science and Engineering Foundation.

\section{References}

1. Sasazuki S, Tamakoshi A, Matsuo K, Ito H, Wakai K, Nagata C, Mizoue T, Tanaka K, Tsuji I, Inoue M and Tsugane S; Research Group for the Development and Evaluation of Cancer Prevention Strategies in Japan: Green tea consumption and gastric cancer risk: an evaluation based on a systematic review of epidemiologic evidence among the Japanese population. Jpn J Clin Oncol 42: 335-346, 2012

2. Yuan JM, Sun C and Butler LM: Tea and cancer prevention: epidemiological studies. Pharmacol Res 64: 123-135, 2011.

3. Stoner GD and Mukhtar H: Polyphenols as cancer chemopreventive agents. J Cell Biochem (Suppl) 22: 169-180, 1995

4. Yoo HG, Shin BA, Park JC, Kim HS, Kim WJ, Chay KO, Ahn BW, Park RK, Ellis LM and Jung YD: Induction of apoptosis by the green tea flavonol (-)-epigallocatechin-3-gallate in human endothelial ECV 304 cells. Anticancer Res 22: 3373-3378, 2002.

5. Jung YD, Kim MS, Shin BA, Chay KO, Ahn BW, Liu W, Bucana CD, Gallick GE and Ellis LM: EGCG, a major component of green tea, inhibits tumour growth by inhibiting VEGF induction in human colon carcinoma cells. Br J Cancer 84: 844-850, 2001.

6. Nagini S: Carcinoma of the stomach: A review of epidemiology, pathogenesis, molecular genetics and chemoprevention. World J Gastrointest Oncol 4: 156-169, 2012.

7. Park JS, Park JH, Khoi PN, Joo YE and Jung YD: MSP-induced RON activation upregulates uPAR expression and cell invasiveness via MAPK, AP-1 and NF-кB signals in gastric cancer cells. Carcinogenesis 32: 175-181, 2011.

8. Feres KJ, Ischenko I and Hayman MJ: The RON receptor tyrosine kinase promotes MSP-independent cell spreading and survival in breast epithelial cells. Oncogene 28: 279-288, 2009.

9. Wang MH, Ronsin C, Gesnel MC, Coupey L, Skeel A, Leonard EJ and Breathnach R: Identification of the ron gene product as the receptor for the human macrophage stimulating protein. Science 266: 117-119, 1994.

10. Chen YQ, Zhou YQ, Angeloni D, Kurtz AL, Qiang XZ and Wang MH: Overexpression and activation of the RON receptor tyrosine kinase in a panel of human colorectal carcinoma cell lines. Exp Cell Res 261: 229-238, 2000.

11. Wang J, Rajput A, Kan JL, Rose R, Liu XQ, Kuropatwinski K, Hauser J, Beko A, Dominquez I, Sharratt EA, Brattain L, Levea C, Sun FL, Keane DM, Gibson NW and Brattain MG: Knockdown of Ron kinase inhibits mutant phosphatidylinositol 3-kinase and reduces metastasis in human colon carcinoma. J Biol Chem 284 10912-10922, 2009.

12. Leonis MA, Thobe MN and Waltz SE: Ron-receptor tyrosine kinase in tumorigenesis and metastasis. Future Oncol 3: 441-448, 2007.

13. Thangasamy A, Rogge J and Ammanamanchi S: Recepteur d'origine nantais tyrosine kinase is a direct target of hypoxiainducible factor-1alpha-mediated invasion of breast carcinoma cells. J Biol Chem 284: 14001-14010, 2009.

14. Lee KE, Park JS, Khoi PN, Joo YE, Lee YH and Jung YD: Upregulation of recepteur d'origine nantais tyrosine kinase and cell invasiveness via early growth response-1 in gastric cancer cells. J Cell Biochem 113: 1217-1223, 2012.
15. Thangasamy A, Rogge $\mathbf{J}$ and Ammanamanchi S: Regulation of RON tyrosine kinase-mediated invasion of breast cancer cells. J Biol Chem 283: 5335-5343, 2008.

16. Zhou D, Pan G, Zheng C, Zheng J, Yian L and Teng X: Expression of the RON receptor tyrosine kinase and its association with gastric carcinoma versus normal gastric tissues. BMC Cancer 8: 353, 2008.

17. Kono S, Ikeda M, Tokudome $\mathrm{S}$ and Kuratsune M: A case-control study of gastric cancer and diet in northern Kyushu, Japan. Jpn J Cancer Res 79: 1067-1074, 1988.

18. Rogers AE, Hafer LJ, Iskander YS and Yang S: Black tea and mammary gland carcinogenesis by 7,12-dimethylbenz(a)anthracene in rats fed control or high fat diets. Carcinogenesis 19: 1269-1273, 1998.

19. Fujiki H, Suganuma M, Okabe S, Sueoka E, Suga K, Imai K, Nakachi K and Kimura S: Mechanistic findings of green tea as cancer preventive for humans. Proc Soc Exp Biol Med 220: 225-228, 1999.

20. Liang YC, Lin-shiau SY, Chen CF and Lin JK: Suppression of extracellular signals and cell proliferation through EGF receptor binding by (-)-epigallocatechin gallate in human A431 epdeidermoid carcinoma cells. J Cell Biochem 67: 55-65, 1997.

21. Kitano K, Nam KY, Kimura S, Fujiki H and Imanishi Y: Sealing effects of (-)-epigallocatechin gallate on protein kinase $\mathrm{C}$ and protein phosphatase 2A. Biophys Chem 65: 157-164, 1997.

22. Fu Y and Chen A: The phyto-chemical (-)-epigallocatechin gallate suppresses gene expression of epidermal growth factor receptor in rat hepatic stellate cells in vitro by reducing the activity of Egr-1. Biochem Pharmacol 72: 227-238, 2006.

23. Moon Y, Lee $M$ and Yang $H$ : Involvement of early growth response gene 1 in the modulation of microsomal prostaglandin $\mathrm{E}$ synthase 1 by epigallocatechin gallate in A549 human pulmonary epithelial cells. Biochem Pharmacol 73: 125-135, 2007.

24. Thiel G and Cibelli G: Regulation of life and death by the zinc finger transcription factor Egr-1. J Cell Physiol 193: 287-292, 2002.

25. Shin SY, Kim JH, Baker A, Lim Y and Lee YH: Transcription factor Egr-1 is essential for maximal matrix metalloproteinase-9 transcription by tumor necrosis factor alpha. Mol Cancer Res 8: 507-519, 2010.

26. Keates S, Keates AC, Nath S, Peek RM Jr and Kelly CP: Transactivation of the epidermal growth factor receptor by $\mathrm{cag}+$ Helicobacter pylori induces upregulation of the early growth response gene Egr-1 in gastric epithelial cells. Gut 54: 1363-1369, 2005.

27. Ma J, Ren Z, Ma Y, Xu L, Zhao Y, Zheng C, Fang Y, Xue T, Sun B and Xiao W: Targeted knockdown of EGR-1 inhibits IL-8 production and IL-8-mediated invasion of prostate cancer cells through suppressing EGR-1/NF-kappaB synergy. J Biol Chem 284: 34600-34606, 2009.

28. Kaufmann K and Thiel G: Epidermal growth factor and plateletderived growth factor induce expression of Egr-1, a zinc finger transcription factor, in human malignant glioma cells. J Neurol Sci 189: 83-91, 2001

29. Cohen DM, Gullans SR and Chin WW: Urea inducibility of egr-1 in murine inner medullary collecting duct cells is mediated by the serum response element and adjacent Ets motifs. J Biol Chem 271: 12903-12908, 1996.

30. Katiyar SK, Afaq F, Azizuddin K and Mukhtar H: Inhibition of UVB-induced oxidative stress-mediated phosphorylation of mitogen-activated protein kinase signaling pathways in cultured human epidermal keratinocytes by green tea polyphenol (-)-epigallocatechin-3-gallate. Toxicol Appl Pharmacol 176: 110-117, 2001.

31. Khan HY, Zubair H, Ullah MF, Ahmad A and Hadi SM: Oral administration of copper to rats leads to increased lymphocyte cellular DNA degradation by dietary polyphenols: implications for a cancer preventive mechanism. Biometals 24: 1169-1178, 2011.

32. Lin JK: Cancer chemoprevention by tea polyphenols through modulating signal transduction pathways. Arch Pharm Res 25: 561-571, 2002.

33. O'Toole JM, Rabenau KE, Burns K, Lu D, Mangalampalli V, Balderes P, Covino N, Bassi R, Prewett M, Gottfredsen KJ, Thobe MN, Cheng Y, Li Y, Hicklin DJ, Zhu Z, Waltz SE, Hayman MJ, Ludwig DL and Pereira DS: Therapeutic implications of a human neutralizing antibody to the macrophage-stimulating protein receptor tyrosine kinase (RON), a c-MET family member. Cancer Res 66: 9162-9170, 2006.

34. Logan-Collins J, Thomas RM, Yu P, Jaquish D, Mose E, French R, Stuart W, McClaine R, Aronow B, Hoffman RM, Waltz SE and Lowy AM: Silencing of RON receptor signaling promotes apoptosis and gemcitabine sensitivity in pancreatic cancers. Cancer Res 70: 1130-1140, 2010. 